NBER WORKING PAPER SERIES

UNDERSTANDING MARKOV-SWITCHING RATIONAL EXPECTATIONS MODELS

\author{
Roger E.A. Farmer \\ Tao Zha \\ Daniel F. Waggoner \\ Working Paper 14710 \\ http://www.nber.org/papers/w14710 \\ NATIONAL BUREAU OF ECONOMIC RESEARCH \\ 1050 Massachusetts Avenue \\ Cambridge, MA 02138 \\ February 2009
}

This paper is a thorough revision of the earlier draft entitled "Understanding the New-Keynesian Model When Monetary Policy Switches Regimes" (NBER Working Paper 12965). We thank the referees and editors for thoughtful comments and Zheng Liu, Richard Rogerson, Eric Swanson, and John Williams for helpful discussions. We are grateful to Jacob Smith for excellent research assistance. This study is supported in part by NSF grant \#0720839. The views expressed herein do not necessarily reflect those of the Federal Reserve Bank of Atlanta nor those of the Federal Reserve System. The views expressed herein are those of the author(s) and do not necessarily reflect the views of the National Bureau of Economic Research.

NBER working papers are circulated for discussion and comment purposes. They have not been peerreviewed or been subject to the review by the NBER Board of Directors that accompanies official NBER publications.

(C) 2009 by Roger E.A. Farmer, Tao Zha, and Daniel F. Waggoner. All rights reserved. Short sections of text, not to exceed two paragraphs, may be quoted without explicit permission provided that full credit, including $\odot$ notice, is given to the source. 
Understanding Markov-Switching Rational Expectations Models

Roger E.A. Farmer, Tao Zha, and Daniel F. Waggoner

NBER Working Paper No. 14710

February 2009

JEL No. C02,C1,E0,E4

\begin{abstract}
$\underline{\text { ABSTRACT }}$
We develop a set of necessary and sufficient conditions for equilibria to be determinate in a class of forward-looking Markov-switching rational expectations models and we develop an algorithm to check these conditions in practice. We use three examples, based on the new-Keynesian model of monetary policy, to illustrate our technique. Our work connects applied econometric models of Markov-switching with forward looking rational expectations models and allows an applied researcher to construct the likelihood function for models in this class over a parameter space that includes a determinate region and an indeterminate region.
\end{abstract}

Roger E.A. Farmer

UC, Los Angeles

Department of Economics

Box 951477

Los Angeles, CA 90095-1477

and NBER

rfarmer@econ.ucla.edu

Tao Zha

Federal Reserve Bank of Atlanta

1000 Peachtree Street N.E.

Atlanta, Georgia 30309-4470

tzha@earthlink.net
Daniel F. Waggoner

Federal Reserve Bank of Atlanta

1000 Peachtree Street N.E.

Atlanta, Georgia 30309-4470

dwaggoner@frbatlanta.org 


\title{
UNDERSTANDING MARKOV-SWITCHING RATIONAL EXPECTATIONS MODELS
}

\author{
ROGER E.A. FARMER, DANIEL F. WAGGONER, AND TAO ZHA
}

\begin{abstract}
We develop a set of necessary and sufficient conditions for equilibria to be determinate in a class of forward-looking Markov-switching rational expectations models and we develop an algorithm to check these conditions in practice. We use three examples, based on the new-Keynesian model of monetary policy, to illustrate our technique. Our work connects applied econometric models of Markov-switching with forward looking rational expectations models and allows an applied researcher to construct the likelihood function for models in this class over a parameter space that includes a determinate region and an indeterminate region.
\end{abstract}

\section{INTRODUCTION}

Reduced form Markov-switching models have been widely used to study economic problems in which there are occasional structural shifts in fundamentals. In an approach initiated by Hamilton (1989), a set of economic time series is modeled as a vector autoregression (VAR) in which the parameters of the process are viewed as the outcome of a discrete state Markov process. It is well known that a constant parameter vector autoregression can be viewed as the reduced form of a forward looking rational expectations model but less is known about the Markov-switching case.

In a recent literature a number of authors have begun to study the relationship between Markov-switching models and forward looking Markov-switching rational expectations (MSRE) models. Work in this area includes papers by Leeper and Zha (2003), Svensson and Williams (2005), Blake and Zampolli (2006), Davig and Leeper

Date: January 30, 2009.

Key words and phrases. Stability, nonlinearity, unique equilibrium, cross-regime indeterminacy, expectations formation, necessary and sufficient conditions.

This paper is a thorough revision of the earlier draft entitled "Understanding the New-Keynesian Model When Monetary Policy Switches Regimes" (NBER Working Paper 12965). We thank the referees and editors for thoughtful comments and Zheng Liu, Richard Rogerson, Eric Swanson, and John Williams for helpful discussions. We are grateful to Jacob Smith for excellent research assistance. This study is supported in part by NSF grant \#0720839. The views expressed herein do not necessarily reflect those of the Federal Reserve Bank of Atlanta nor those of the Federal Reserve System. 
(2006, 2007), and Farmer, Waggoner, and Zha (2008a). MSRE models are more complicated than linear rational expectations models since the agents of the model must be allowed to take account of the possibility of future regime changes when forming expectations.

To make progress with empirical work that uses the MSRE approach one must be able to write down the likelihood function for a complete class of possible solutions. In the case of linear models, Lubik and Schorfheide (2003) have shown how to partition the parameter space into two disjoint regions: one in which there exists a unique determinate rational expectations equilibrium and one in which there exist multiple indeterminate solutions driven by non-fundamental shocks. One would like to be able to find a similar partition for the case of MSRE models but, in order to accomplish this task, one would need to find a set of necessary and sufficient conditions under which an MSRE model has a unique determinate solution. This paper provides such conditions for an important subset of MSRE models; those in which there are no predetermined variables.

Our paper is structured in the following way. In Section II we discuss the relationship of our paper to previous literature. Section III introduces the class of forward looking Markov-switching rational expectations models that we will study and Section IV reviews known results for the linear model. In Section V we discuss some results from the engineering literature and explain the differences between alternative stability concepts that are equivalent in linear models but different in Markov-switching systems. Sections VI and VII contain our main results; a characterization theorem and a set of necessary and sufficient conditions for determinacy of equilibrium. In Section VIII we provide an algorithm that is straightforward to apply in practice and in Section IX we apply our results to a familiar example; that of the new-Keynesian model of monetary policy. Section X presents some concluding comments.

\section{RElAted Literature}

Markov switching models in economics were first discussed by Hamilton (1989) who applied them to autoregressive models of gdp where the parameters of the model are allowed to switch between two regimes. Forward-looking regime switching models have been studied by Svensson and Williams (2005), Davig and Leeper (2006, 2007) and Farmer, Waggoner, and Zha (2008a,b,c), who use them to study the effectiveness of monetary policy. We briefly review the issues that arise in that literature to explain why the current paper has relevance to an important body of applied research and the debate over the causes of an observed reduction in the volatility of macroeconomic 
variables in the period after 1980 - a phenomenon widely referred to as the Great Moderation.

In the context of this debate, Sims and Zha (2006) use a backward-looking Markovswitching model to ask: Were there regime changes in US monetary policy? Their preferred explanation for the Great Moderation is that it was caused by changes to the shock variances of an identified vector autoregression. An alternative explanation, due to Cogley and Sargent $(2002,2005)$, argues that changes in observed behavior of US time series is due to parameter drift in a random coefficient model.

Clarida et. al. (2000) and Lubik and Schorfheide (2004) have presented a third view. They argue that the policy followed by the Fed before 1980 led to indeterminate equilibria that permitted non-fundamental 'sunspot' shocks to add volatility to realized outcomes. Although this explanation for the Great Moderation is intriguing, it is inconsistent with the rational expectations assumption: If policy has switched in the past, it might be expected to switch again in the future. Agents in the model studied by these authors do not take account of this possibility.

The papers of Svensson and Williams (2005), Davig and Leeper (2006, 2007) and Farmer, Waggoner, and Zha (2008a,b,c) extend the class of models studied by Clarida et. al. and Lubik and Schorfheide to the Markov-switching rational expectations environment. This extension is important because it connects the reduced form econometric literature with structural economic theory and allows investigators to account for anticipation effects. In this environment it becomes possible to ask the question: Was the Great Moderation caused by a change in the parameters of the policy rule in a structural model or by a reduction in the variance of structural disturbances?

Although the MSRE literature has made some headway in addressing questions like this there has been, until now, no known set of necessary and sufficient conditions to determine if the parameters of a Markov-switching rational expectations model lead to a determinate equilibrium. Davig and Leeper (2007) show that some solutions to the MSRE model have a linear representation and they find conditions for the solution to this linear representation to be unique; but Farmer, Waggoner, and Zha (2008a) show that these conditions do not apply to the original Markov-switching rational expectations model.

In the current paper we provide a complete set of necessary and sufficient conditions for a large class of forward looking MSRE models to be determinate. Our results provide the necessary tools for applied researchers to estimate structural models in this class using maximum likelihood methods. 


\section{The Class of Models}

We study a class of ergodic multivariate forward-looking rational expectations models in which the parameters follow a discrete state Markov chain indexed by $s_{t}$ with transition matrix $P=\left[p_{i j}\right]$. The element $p_{i j}$ represents the probability that $s_{t}=j$ given $s_{t-1}=i$ for $i, j \in\{1, \ldots h\}$ where $h \geq 1$ is the number of regimes and when $s_{t}=i$ we say that the system is in regime $i{ }^{1}$ The models we study are represented by the equation,

$$
\Gamma_{s_{t}} y_{t}=E_{t} y_{t+1}+\Psi_{s_{t}} u_{t}
$$

where $y_{t}$ is an $n$-dimensional vector of endogenous random variables with finite first and second moments, $\Gamma_{s_{t}}$ is an invertible $n \times n$ matrix, $\Psi_{s_{t}}$ is an $n \times m$ matrix, and $u_{t}$ is an $m$-dimensional vector of exogenous shocks that are assumed to be stationary. While the existence of a solution to Equation (1) depends on the properties of $u_{t}$, its uniqueness does not. Thus, to simplify the exposition, we assume without loss of generality that $u_{t}$ is iid, mean-zero, and independent of the Markov process $s_{t}$.

We interpret $y_{t}$ to be a vector of economic variables that depends on expectations of its own future value and we seek a solution to Equation (1) that satisfies a suitable stability concept.

\section{The Linear CASE}

To explain our approach, we will spend some time discussing the familiar case when $h=1$ for which Equation (1) is linear and can be written as follows,

$$
\Gamma y_{t}=E_{t} y_{t+1}+\Psi u_{t}
$$

In this case a solution is a stable stochastic process that satisfies Equation (2). Depending on the values of the parameters there may be one or more solutions.

One solution, referred to as a minimal state variable (MSV) solution following McCallum (1983), describes $y_{t}$ as a linear function of the fundamental shocks $\left\{u_{t}\right\}$. For Equation (2), a solution of this kind exists and is given by the expression,

$$
y_{t}=G u_{t}
$$

where

$$
G=\Gamma^{-1} \Psi
$$

\footnotetext{
${ }^{1}$ The engineering literature (Costa, Fragoso, and Marques, 2004) uses mode to refer to what we call a regime.
} 
We require a solution to Equation (2) to be stable because economic agents are assumed to base decisions on expectations of the future values of $y_{t}$ and these expectations are obtained by recursively iterating Equation (2) into the future; stability ensures that this process is well defined.

For some parameter configurations, and some definitions of stability, there may be an infinite set of solutions to Equation (2) all of which are stable. When this occurs, each member of the set is said to be an indeterminate equilibrium. The minimal state variable solution is a member of this set but there may be other solutions that are serially correlated and add additional volatility to the time paths of the state variables.

In two recent papers on the empirical importance of indeterminate equilibria, Lubik and Schorfheide $(2003,2004)$ show how to write an indeterminate solution as a linear combination of the minimal state variable solution and a first order moving average component. These solutions can be written as follows,

$$
\begin{aligned}
y_{t} & =G u_{t}+w_{t}, \\
w_{t} & =\Lambda w_{t-1}+V \gamma_{t} .
\end{aligned}
$$

In these expressions, $\gamma_{t}$ is a stable, $k$-dimensional, zero-mean, non-fundamental disturbance that may or may not be correlated with the fundamental shock $u_{t}$, $k$ is the number of eigenvalues of $\Gamma$ that are inside the unit circle and $\Lambda$ is an $n \times n$ matrix of rank $k$, of the form

$$
\Lambda=V \Phi V^{\prime}
$$

with

$$
r_{\sigma}(\Phi)<1
$$

The notation $r_{\sigma}(\Phi)$ denotes the spectral radius of $\Phi$, which is the maximum of the absolute value of the eigenvalues of $\Phi$. The $n \times k$ matrix $V$ has orthonormal columns and the $k \times k$ matrix $\Phi$ is block upper triangular and its eigenvalues are the stable eigenvalues of $\Gamma$. Equation (7) is equivalent to

$$
\Gamma V=V \Phi .
$$

Note that it is not true in general that $\Gamma=V \Phi V^{\prime}$ since $\Phi$ contains only a subset of the eigenvalues of $\Gamma$. Both the matrices $\Phi$ and $V$ can be easily obtained from the real Schur decompostion of $\Gamma$.

There are two important lessons to be learned from the linear model. First, by writing solutions in the form of Equations (5) and (6) it is possible to convert the question of whether there is a unique determinate solution to Equation (2) into the 
related question of whether Equation (6) is a stable stochastic process. Second, to answer the determinacy question we must settle on a suitable concept of stability.

In the following section we will define two concepts: mean-square stability and bounded stability. These concepts are equivalent in the linear model but, in models with Markov-switching, they are no longer the same. We will explain why engineers chose mean-square stability as the appropriate stability concept over bounded stability and discuss some lessons that can be learned from the engineers.

\section{What ENGINEERING HAS TO TEACH US}

Our strategy for finding necessary and sufficient conditions for indeterminacy is to show that solutions to Equation (1) have a similar representation to the moving average solutions, Equations (5) and (6), that solve the linear model. This turns the determinacy question into one of stability and allows us to appeal to theorems from the engineering literature on the existence and uniqueness of stable solutions to a class of equations that economists call Markov-switching models and engineers refer to as discrete-time Markov jump linear systems. ${ }^{2}$ These are VARs in which the parameters are governed by a discrete state Markov chain and they can be represented by the following expression,

$$
x_{t}=A_{s_{t}} x_{t-1}+B_{s_{t}} \xi_{t},
$$

where $x_{t}$ is an $n$-dimensional stochastic process, $A_{s_{t}}$ is an $n \times n$ matrix, $B_{s_{t}}$ is an $n \times m$ matrix, and $\xi_{t}$ is a stable $m$-dimensional process independent of the Markov process $s_{t}$.

Our main idea is to show that all solutions to Equation (1) can be written as the sum of two particular solutions, one of which depends only on the current regime and the other is a Markov-switching system with the same form as Equation (10). We are thus able to convert the question of whether Equation (1) has a unique determinate solution to the equivalent question of whether Equation (10) possesses a unique stable solution. This approach requires that we define what it means for the solution to a Markov-switching model to be stable.

The Markov-switching system described by Equation (10) is mean-square stable if its first and second moments converge to well defined limits as the horizon extends to infinity. If, in addition, the process is also bounded, then we say it is boundedly stable. The formal definitions of mean-square stability and bounded stability are given below.

\footnotetext{
${ }^{2}$ Since there is a large economics literature that uses the term Markov-switching system, we will use the prevailing economic terminology from this point on.
} 
Definition 1. An $n$-dimensional process $x_{t}$ is mean-square stable (MSS) if and only if there exists an $n$-vector $\mu$ and an $n \times n$ matrix $\Sigma$ such that

(a) $\lim _{t \rightarrow \infty} E_{0}\left[x_{t}\right]=\mu$,

(b) $\lim _{t \rightarrow \infty} E_{0}\left[x_{t} x_{t}^{\prime}\right]=\Sigma$.

Definition 2. An $n$-dimensional process $x_{t}$ is bounded if there exists a real number $N$ such that

$$
\left\|x_{t}\right\|<N \text {, for all } t,
$$

where $\|\cdot\|$ is any well-defined norm. If, in addition, the process is MSS, then the process is said to be boundedly stable.

Other notions of stability could be used in place of mean-square stability. For instance, in economics covariance stationarity is often used, or asymptotic covariance stationarity can be used if one wishes to avoid taking a stand on initial conditions. ${ }^{3}$ In general, asymptotic covariance stationarity is strictly stronger than mean-square stability. For the system given by Equation (10), however, under the assumption that the innovation process $\xi_{t}$ is asymptotically covariance stationary, the system will mean-square stable if and only if it is asymptotically covariance stationary. Because many of the standard theorems we use in this paper are stated in terms of meansquare stability, we also use mean-square stability, but the reader should note that the results of our paper would hold if asymptotic covariance stationarity were used instead. Throughout this paper, we shall use the terms stability and mean-square stability interchangeably.

For linear systems with bounded shocks, mean-square stability and bounded stability are equivalent concepts for determining uniqueness of the equilibrium. For Markov-switching models, however, these two concepts are not the same and one must choose between them. Engineers use mean-square stability for several reasons. First, there are many instances of engineering problems in which the system may be unstable in one or more of its regimes. But as long as this regime does not occur too frequently the state variables will still converge to a well defined ergodic distribution with finite first and second moments. Unstable regimes would be ruled out by bounded stability and this definition of stability would define many interesting physical phenomena to be unstable even though they possess well behaved limiting distributions. Second, most practical applications assume that the system is driven by unbounded errors; for example, normal or lognormal distributions are frequently

\footnotetext{
${ }^{3}$ Some authors use the terms "wide-sense stationarity" and "asymptotic wide-sense stationarity" instead of "covariance stationarity" and "asymptotic covariance stationarity."
} 
assumed, and hence the state variables are also unbounded in practical applications. Third, bounded stability is difficult to work with and there is no known set of necessary and sufficient conditions under which a Markov-switching system will display stability in this stronger sense.

All three of these issues arise in economics. Economics, like engineering, is ripe with examples where one or more regimes are unstable. For example, hyperinflation in Argentina in the 1980's and 1990's was characterized by a series of explosive regimes that were subsequently stabilized. Second, although economic theorists often use boundedness as a stability concept, applied researchers typically use shock distributions with unbounded errors. Finally, to see why bounded stability poses a practical difficulty, consider the following example from (Costa, Fragoso, and Marques, 2004, page 39).

Consider a two dimensional system

$$
x_{t}=A_{s_{t}} x_{t-1},
$$

where $A_{i}$ is a $2 \times 2$ matrix that take the following values,

$$
A_{1}=\left[\begin{array}{cc}
0 & 2 \\
0 & 0.5
\end{array}\right] \text { and } A_{2}=\left[\begin{array}{cc}
0.5 & 0 \\
2 & 0
\end{array}\right] \text {, }
$$

and consider the following two alternative transition matrices,

$$
\left[\begin{array}{ll}
0.8 & 0.2 \\
0.4 & 0.6
\end{array}\right] \text { and }\left[\begin{array}{cc}
0.9 & 0.1 \\
0.4 & 0.6
\end{array}\right] \text {. }
$$

In this example the roots of $A_{1}$ and $A_{2}$ both lie inside the unit circle and if the state were to remain in either regime 1 or regime 2 the system would be stable. Although both roots of $A_{1}$ and both roots of $A_{2}$ are inside the unit circle, the product $A_{1} A_{2}$ has a root outside the unit circle. This means that as long as the system alternates between regime 1 and 2 , the process $x_{t}$ would grow exponentially and hence the system is unbounded regardless of the values of the transition matrix.

In general, for a process given by Equation (11) to be bounded, all roots of all possible products of $A_{1}$ and $A_{2}$ must be inside the unit circle. For boundedness to be a workable stability concept one would need to find a simple condition under which all possible possible products of the coefficient matrices have roots inside the unit circle and to our knowledge, no such condition is known.

Although there is no known way to check for bounded stability, mean-square stability is much easier to deal with and Costa et. al. (2004, pages 34-36) show that 
mean-square stability of (11) is equivalent to the question: Are all roots of the matrix

$$
\left[\begin{array}{ll}
p_{1,1} A_{1} \otimes A_{1} & p_{2,1} A_{2} \otimes A_{2} \\
p_{1,2} A_{1} \otimes A_{1} & p_{2,2} A_{2} \otimes A_{2}
\end{array}\right]
$$

inside the unit circle? For the first transition matrix given in (13), the matrix expressed in (14) has a root outside the unit circle and so the system is unstable even though it is stable in each regime separately, a somewhat surprising result. On the other hand, for the second transition matrix given in (13), all the roots of the matrix expressed in (14) are inside the unit circle and hence the system is stable. These results illustrate that mean-square stability and bounded stability are different stability concepts.

\section{A Definition and a Characterization Theorem}

In this section we move beyond the engineering literature. Our main result is to show that the complete set of solutions to the MSRE model, Equation (1), can be described as the sum of two particular solutions. One is what McCallum (1983) has called a minimal state variable solution (MSV) and the other is a Markov-switching system.

We begin by defining a rational expectations equilibrium and proving a theorem that characterizes a class of stochastic processes that satisfy this definition.

Definition 3. A rational expectations equilibrium is a mean-square stable stochastic process that satisfies Equation (1).

The following theorem characterizes all possible solutions to Equation (1), whether or not they satisfy mean-square stability. ${ }^{4}$

Theorem 1. Any solution to the MSRE model (1) can be written in the following way:

$$
\begin{aligned}
y_{t} & =G_{s_{t}} u_{t}+w_{t} \\
w_{t} & =\Lambda_{s_{t-1}, s_{t}} w_{t-1}+V_{s_{t}} V_{s_{t}}^{\prime} \gamma_{t}
\end{aligned}
$$

where $V_{s_{t}}$ is an $n \times k_{s_{t}}$ matrix with orthonormal columns and $0 \leq k_{s_{t}} \leq n, \gamma_{t}$ is an arbitrary $n$-dimensional shock process such that $E_{t-1}\left[V_{s_{t}} V_{s_{t}}^{\prime} \gamma_{t}\right]=0, \Lambda_{s_{t-1}, s_{t}}$ is an

\footnotetext{
${ }^{4}$ This includes solutions from our previous work Farmer, Waggoner, and Zha (2008a,b) which, superficially, appear to have a different form.
} 
$n \times n$ matrix of the form $V_{s_{t}} \Phi_{s_{t-1}, s_{t}} V_{s_{t-1}}^{\prime}$ for some $k_{s_{t}} \times k_{s_{t-1}}$ matrix $\Phi_{s_{t-1}, s_{t}}$ such that

$$
\Gamma_{i} V_{i}=\sum_{j=1}^{h} p_{i, j} V_{j} \Phi_{i, j} \text { for } 1 \leq i \leq h,
$$

and $G_{s_{t}} u_{t}$ is the minimum-state-variable (MSV) solution with $G_{s_{t}}=\Gamma_{s_{t}}^{-1} \Psi_{s_{t}}$.

Proof. See Appendix A.

This theorem states that all solutions to the MSRE model can be written in an analogous form to the Lubik-Schorfheide representation of solutions to the linear system; recall that these were represented as the sum of a minimal state variable solution and a moving average component. Our contribution is to show that, in the case of MSRE models, the moving average component is a Markov-switching system and it is this theorem that allows us to appeal to results from the engineering literature to find conditions for the model defined by Equation (1) to be determinate.

The most important part of our result is Equation (17), which plays the role of Equation (9) for the linear system. Whereas the matrices $\Phi$ and $V$ in Equation (9) can be obtained directly from the real Schur decomposition of $\Gamma$, techniques to find the set of matrices $\Phi_{i, j}$ and $V_{i}$ are more involved. As we will see in the next section, Equation (17) will be key in devising an algorithm for determining whether or not there are multiple stable solutions to Equation (1).

\section{NeCEssary And Sufficient Conditions for a Unique Equilibrium}

In this section we develop a set of necessary and sufficient conditions for the existence of a unique rational expectations equilibrium. We assume that the sunspot random process $\gamma_{t}$ has mean zero, is mean-square stable, and is independent of the fundamental Markov process $s_{t}$. We introduce the following definition:

$$
M_{1}\left(X_{i, j}\right)=\left[\begin{array}{ccc}
p_{1,1} X_{1,1} \otimes X_{1,1} & \cdots & p_{h, 1} X_{h, 1} \otimes X_{h, 1} \\
\vdots & \ddots & \vdots \\
p_{1, h} X_{1, h} \otimes X_{1, h} & \cdots & p_{h, h} X_{h, h} \otimes X_{h, h}
\end{array}\right] .
$$

The matrices $M_{1}\left(\Lambda_{i, j}\right)$ and $M_{1}\left(\Phi_{i, j}\right)$ are important because they play a role in ex-

pressing the variance of $w_{t}$ in terms of the variance of $w_{t-1}$. The details of this are outlined in the proof of the following theorem.

Theorem 2. The process represented by Eqs (15)-(17) is a mean-square stable solution to the MSRE model (1) if and only if

$$
r_{\sigma}\left(M_{1}\left(\Phi_{i, j}\right)\right)<1
$$


Proof. See Appendix A.

Since a rational expectation equilibrium is defined as a mean-square stable solution to Equation (1), Theorem 2 provides necessary and sufficient conditions for determinacy; that is, for the rational expectations equilibrium to be unique. In the linear case the analogous conditions can easily be checked using the real Schur decomposition of a known matrix. For the Markov-switching case, however, the conditions provided in Theorem 2 are difficult to verify in practice because finding $V_{i}$ and $\Phi_{i, j}$ that satisfy Equations (15) - (17) is not a standard problem in matrix algebra. It is, however, equivalent to a collection of constrained optimization problems.

For each choice of dimensions $\left\{k_{1}, \cdots, k_{h}\right\}$, Equation (17) and the orthogonality conditions on the columns of $V_{i}$ provide constraints on the $V_{i}$ and $\Phi_{i, j}$. Subject to these constraints, one minimizes the objective function $r_{\sigma}\left(M_{1}\left(\Phi_{i, j}\right)\right)$. If the minimum value of the objective function is less than one, then the $V_{i}$ and $\Phi_{i, j}$ that give the minimun define a MSS solution different from the MSV solution. On the other hand, if for all choices of dimensions $\left\{k_{1}, \cdots, k_{h}\right\}$, not all zero, the minimum value is greater than or equal to one, then there can be no MSS solution other than the MSV solution. This is formalized in the following corollary.

Corollary 1. Let $0 \leq k_{i} \leq n$. Consider the problem of choosing $n \times k_{i}$ matrices $V_{i}$ and $k_{j} \times k_{i}$ matrices $\Phi_{i, j}$ such that $r_{\sigma}\left(M_{1}\left(\Phi_{i, j}\right)\right)$ is minimized subject to the constraints $\Gamma_{i} V_{i}=\sum_{j=1}^{h} p_{i, j} V_{j} \Phi_{i, j}$ and $V_{i}^{\prime} V_{i}=I_{k_{i}}$.

(1) If there exists some choice of $\left\{k_{1}, \cdots, k_{h}\right\}$, not all zero, such that the optimal solution $r_{\sigma}\left(M_{1}\left(\Phi_{i, j}\right)\right)$ is smaller than one, there will be multiple solutions to Equation (1).

(2) If, for all possible choices of $\left\{k_{1}, \cdots, k_{h}\right\}$, not all zero, the minimum value of $r_{\sigma}\left(M_{1}\left(\Phi_{i, j}\right)\right)$ is greater than or equal to one, there will be only one meansquare stable solution to the MSRE model (1). This solution is the MSV solution.

Proof. The proof follows directly from Theorem 2.

\section{ViII. Constructing Sunspot Solutions}

While Theorem 2 and its corollary provide a general technique for determining if there is a unique MSS solution of Equation (1), we consider a special case where we are able to explicitly solve the constraints and thus characterize this class of solutions. Given the complexity of the problem, this illustrative case is important because it allows one to develop intuition about MSS solutions other than the MSV solution and 
relate our solution technique to the standard eigenvalue problem. It is also a case with practical relevance as we demonstrate in Section IX.

The case we consider is that of $k_{i}=1$ for $1 \leq i \leq h$. This implies that $V_{i}$ will be a vector and $\Phi_{i, j}$ will be a scalar. The following proposition characterizes solutions of this form.

Proposition 1.

(1) If there exist scalars $c_{1}, \cdots, c_{h}$ and an $n h$-dimensional vector $v=\left(v_{1}, \cdots, v_{h}\right)$ with $v_{i} \neq 0$ such that

$$
\begin{gathered}
\left(\operatorname{diag}\left(\Gamma_{i}\right)-\left(\left(\operatorname{diag}\left(c_{i}\right) P\right) \otimes I_{n}\right)\right) v=0, \\
r_{\sigma}\left(\operatorname{diag}\left(c_{i}^{2}\right) P\right)<1
\end{gathered}
$$

then there exist mean-square stable solutions to Equation (1), other than the MSV solution.

(2) These solutions are given by Equations (15) and (16) where

$$
\begin{aligned}
V_{s_{t}} & =\frac{v_{s_{t}}}{\left\|v_{s_{t}}\right\|}, \\
\Lambda_{s_{t-1}, s_{t}} & =\frac{c_{s_{t-1}}}{\left\|v_{s_{t-1}}\right\|^{2}} v_{s_{t}} v_{s_{t-1}}^{\prime} .
\end{aligned}
$$

(3) The solutions defined in part (2) are bounded if and only if $\left|c_{i}\right|<1$.

Proof. See Appendix A.

Part (3) of this proposition gives a complete characterization of the set of boundedly stable solutions of this form. Recall that in general one would need to check if all possible permutations of all possible products of matrices have roots inside the unit circle. This is simple in the case of $k_{i}=1$ since the relevant matrices become scalars and scalar multiplication is commutative.

The proposition allows us to check for mean-square stable solutions by solving the nonlinear equation

$$
\operatorname{det}\left(\operatorname{diag}\left(\Gamma_{i}\right)-\left(\left(\operatorname{diag}\left(c_{i}\right) P\right) \otimes I_{n}\right)\right)=0
$$

and seeing if Equation (21) holds. In the case of one regime, this optimization problem reduces to checking to see if $\Gamma$ has an eigenvalue inside the unit circle.

In the case of two or more regimes the numbers $c_{i}$ are analogous to eigenvalues, and are eigenvalues if all the $c_{i}$ are forced to be equal. However, in general, there will be a continuum of solutions of Equation (24). For example, when $h=2$, Equation 
(24) defines a pair of curves $c_{1}=\psi\left(c_{2}\right)$, which correspond to the two branches of the solution. The question of determinacy amounts to asking whether the correspondence $c_{1}=\psi\left(c_{2}\right)$ has an intersection with the region defined by the spectral radius condition, Equation (21). In Section IX, we provide economic examples and plot the correspondence $c_{1}=\psi\left(c_{2}\right)$ for each example. We provide one example where there is a unique solution and two examples where there is a continuum of solutions for which the spectral radius condition is satisfied.

\section{An Application to the New-Keynesian Model}

In this section, we apply our theoretical results to the canonical new-Keynesian model studied by Lubik and Schorfheide $(2004) ;^{5}$

$$
\begin{aligned}
\text { AS curve } & \pi_{t}=\beta E_{t} \pi_{t+1}+\kappa x_{t}+u_{t}^{S}, \\
\text { IS curve } & x_{t}=E_{t} x_{t+1}-\sigma^{-1}\left(i_{t}-E_{t} \pi_{t+1}\right)+u_{t}^{D}, \\
\text { Policy rule } & i_{t}=\alpha_{s_{t}} \pi_{t}+\iota_{s} x_{t},
\end{aligned}
$$

where $x_{t}$ is the deviation of output from its trend path, $\pi_{t}$ is a percentage deviation from its steady state value, $i_{t}$ is the nominal interest rate, $u_{t}^{D}$ is an aggregate demand shock, $u_{t}^{S}$ is an aggregate supply shock and we allow for both both $u_{t}^{D}$ and $u_{t}^{S}$ to be serially correlated:

$$
\begin{aligned}
& u_{t}^{S}=\rho_{S} u_{t-1}^{S}+\varepsilon_{t}^{S}, \\
& u_{t}^{D}=\rho_{D} u_{t-1}^{D}+\varepsilon_{t}^{D} .
\end{aligned}
$$

The innovations $\varepsilon_{t}^{S}$ and $\varepsilon_{t}^{D}$ are stationary exogenous random processes satisfying $E_{t} \varepsilon_{t+1}^{S}=E_{t} \varepsilon_{t+1}^{D}=0$.

The private sector block, consisting of Equations (25) and (26), has three regimeindependent parameters, $\sigma, \beta$ and $\kappa$. The parameter $\sigma$ represents the intertemporal elasticity of substitution, $\beta$ is the discount factor of the representative household, and $\kappa$ is the slope of the Phillips curve. Uncertain monetary policy, represented by Equation (27), has two regime-dependent parameters, $\alpha_{s_{t}}$ and $\iota_{s_{t}}$, that capture the degree to which monetary policy is active or passive and we concentrate on the case of two regimes by setting $h=2$.

\footnotetext{
${ }^{5}$ Liu, Waggoner, and Zha (2008) show how to derive this MSRE model directly from the consumers and firms' optimization problems.
} 
To write the new-Keynesian model in compact form, we substitute Equation (27) into Equation (26). Rearranging terms the model can then be written as

$$
F_{s_{t}} y_{t}=H E_{t} y_{t+1}+u_{t}
$$

where

$$
\begin{array}{rlrl}
y_{t} & =\left[\begin{array}{l}
\pi_{t} \\
x_{t}
\end{array}\right], & u_{t}=\left[\begin{array}{c}
u_{t}^{S} \\
u_{t}^{D}
\end{array}\right], \\
F_{s_{t}}=\left[\begin{array}{cc}
1 & -\kappa \\
\sigma^{-1} \alpha_{s_{t}} & 1+\sigma^{-1} \iota_{s_{t}}
\end{array}\right], & H=\left[\begin{array}{cc}
\beta & 0 \\
\sigma^{-1} & 1
\end{array}\right] .
\end{array}
$$

This is a special case of Equation (1) where $\Gamma_{s_{t}}=H^{-1} F_{s_{t}}$ and $\Psi_{s_{t}}=H^{-1}$.

The solution to this new-Keynesian model has the same form as Equations (15) and (16) but because we allow for autocorrelated errors, the coefficient matrices $G_{s_{t}}$ (for $s_{t}=1, \ldots, h$ ) for the MSV solution have the following form:

$$
\operatorname{vec}\left[\begin{array}{c}
G_{1} \\
\vdots \\
G_{h}
\end{array}\right]=\left[I_{m} \otimes \operatorname{diag}\left(\Gamma_{i}\right)-\rho^{\prime} \otimes P \times I_{n}\right]^{-1} \text { vec }\left[\begin{array}{c}
\Psi_{1} \\
\vdots \\
\Psi_{h}
\end{array}\right],
$$

where $\rho$ is a $2 \times 2$ diagonal matrix whose diagonal vector is $\left[\rho_{S}, \rho_{D}\right]$. For this model we can make use of Proposition 1 to find values of $c_{1}$ and $c_{2}$ such that $r_{\sigma}<1$.

Following Leeper (1991), the literature on Taylor Rules defines a regime in which the interest rate is changed by more than one for one in response to a change in expected inflation, to be an active regime. If the interest rate responds less than one for one, the regime is said to be passive. The response coefficient of the Fed is represented by the parameter $\alpha$ and the fact that regime 1 is passive and regime 2 is active is represented in our model by setting $\left|\alpha_{1}\right|<1$ and $\left|\alpha_{2}\right|>1$. The matrix $P$ determines the persistence of each regime.

We will provide three examples, one example with a unique determinate equilibrium and two examples with a continuum of indeterminate equilibria and we will illustrate Proposition 1 in graphs. For all the examples we choose the following parameter values:

$$
\begin{gathered}
\alpha_{1}=0.8, \iota_{1}=0.0, \iota_{2}=0.0 \text { (regime dependent parameters); } \\
\beta=0.99, \sigma=1.0, \kappa=0.132, \rho_{S}=\rho_{D}=0.9 \text { (private-sector parameters); } \\
p_{22}=0.95 \text { (probability of staying in the second regime). }
\end{gathered}
$$

Whether a model is determinate or not is a complicated question and the answer depends on many parameter values in the model. In this section, we vary the values 


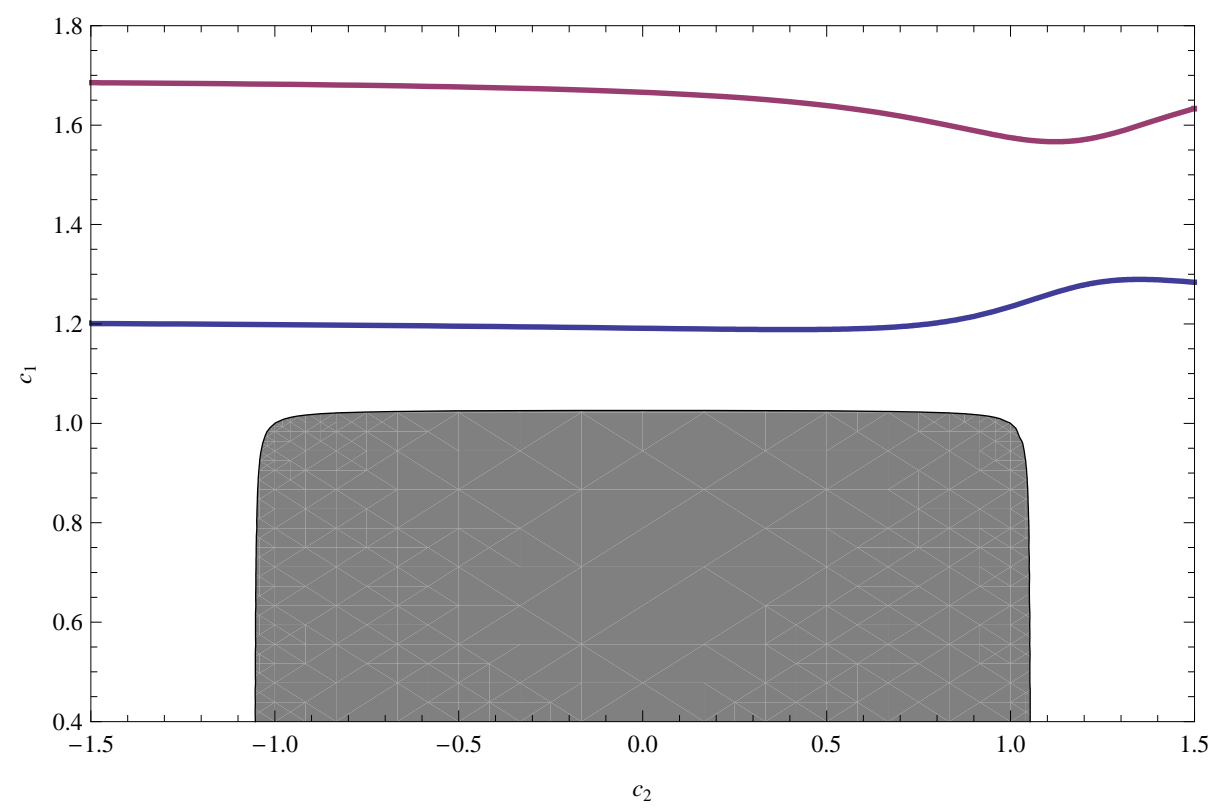

Figure 1. The new-Keynesian model with the baseline parameterization: $\alpha_{1}=0.8, \alpha_{2}=2.5, \iota_{1}=\iota_{2}=0.0, \beta=0.99, \sigma=1.0, \kappa=$ $0.132, p_{11}=0.75$, and $p_{22}=0.95$. The two lines are defined by Equation (24) and the shaded region is defined Inequality (21).

of only $\alpha_{2}$ and $p_{11}$ and focus on an illustration which demonstrates that uniqueness of the equilibrium can be affected by the degree of an active monetary policy and by the persistence of the active regime relative to the passive regime.

IX.1. Example 1. In this example, we set

$$
\alpha_{2}=2.5, p_{11}=0.75
$$

For this parameterization, one can verify that part (2) in Corollary 1 is satisfied. Thus, the equilibrium is unique and characterized by the MSV solution, whose coefficient matrices are given by Equation (31).

Figure 1 gives an intuitive explanation of why the equilibrium is unique. Given the parameter values in this example, we compute the values $c_{1}=\psi\left(c_{2}\right)$ for every value of $c_{2}$. The correspondence $c_{1}=\psi\left(c_{2}\right)$ has two branches for a given value of $c_{2}$ : we plot both branches on the figure as two lines both of which define values of $c_{1}$ and $c_{2}$ that satisfy Equation (20). We also compute the region defined by Inequality (21). Figure 1 shows that for this case there is an empty intersection of the correspondence with the region and hence there are no stable equilibria other than the MSV solution. All values of the correspondence $c_{1}=\psi\left(c_{2}\right)$ represent solutions to Equation (1) that are unstable and hence are ruled out by Definition 3. 
If there were no switching between regimes, regime 1 would be associated with an indeterminate equilibrium and regime 2 would be associated with a unique determinate equilibrium. This follows from the fact that for our chosen parameters, the matrix $\Gamma_{1}$ has a root inside the unit circle whereas $\Gamma_{2}$ has both roots outside. When there is Markov switching between regimes, it is the set of regimes that is determinate.

IX.2. Example 2. In this example, we set

$$
\alpha_{2}=1.05, p_{11}=0.75
$$

This example differs from the previous example only in that $\alpha_{2}$ is smaller. Even with this smaller value of $\alpha_{2}$, regime 2 by itself would be associated with a unique determinate equilibrium because $\Gamma_{2}$ has both roots outside the unit circle. We now use this example to demonstrate that the magnitude of an active policy's response to inflation plays an essential role in determining uniqueness of the equilibrium. The active policy in this case is too weak to ensure a unique equilibrium, as shown in Figure 2. Recall that the correspondence $c_{1}=\psi\left(c_{2}\right)$ shows values of $c_{1}$ and $c_{2}$ that satisfy Equation (20) and shaded region represents points that satisfy Inequality (21). Using part (1) of Proposition 1; the fact that the lower branch of the correspondence intersects with the upper right corner shaded region implies that there is a continuum of indeterminate stable equilibria.

IX.3. Example 3. A third example is provided by the following parameterization.

$$
\alpha_{2}=1.05, p_{11}=0.90 \text {. }
$$

Here, the duration of regime 1 relative to regime 2 is longer than that in example 2. As discussed above, regime 1 in isolation would be associated with an indeterminate equilibrium. We saw in example 2 that there was only a small set of values of $c_{1}=\psi\left(c_{2}\right)$ and $c_{2}$ that were associated with mean-square stable equilibria. Figure 3 demonstrates that, when the passive regime is more persistent, the lower branch of the correspondence $c_{1}=\psi\left(c_{2}\right)$ intersects with a much larger portion of the shaded region than in example 2 ; hence there is a larger set of values of $c_{1}$ and $c_{2}$ for which there exists an indeterminate equilibrium.

Example 3 is interesting not only because indeterminacy persists for a wider range of parameter values but also because the characteristics of the indeterminate solutions in this case display an unexpected property; the persistence of shocks in the active regime can take a wide range of values. The parameters $c_{1}$ and $c_{2}$ determine the 


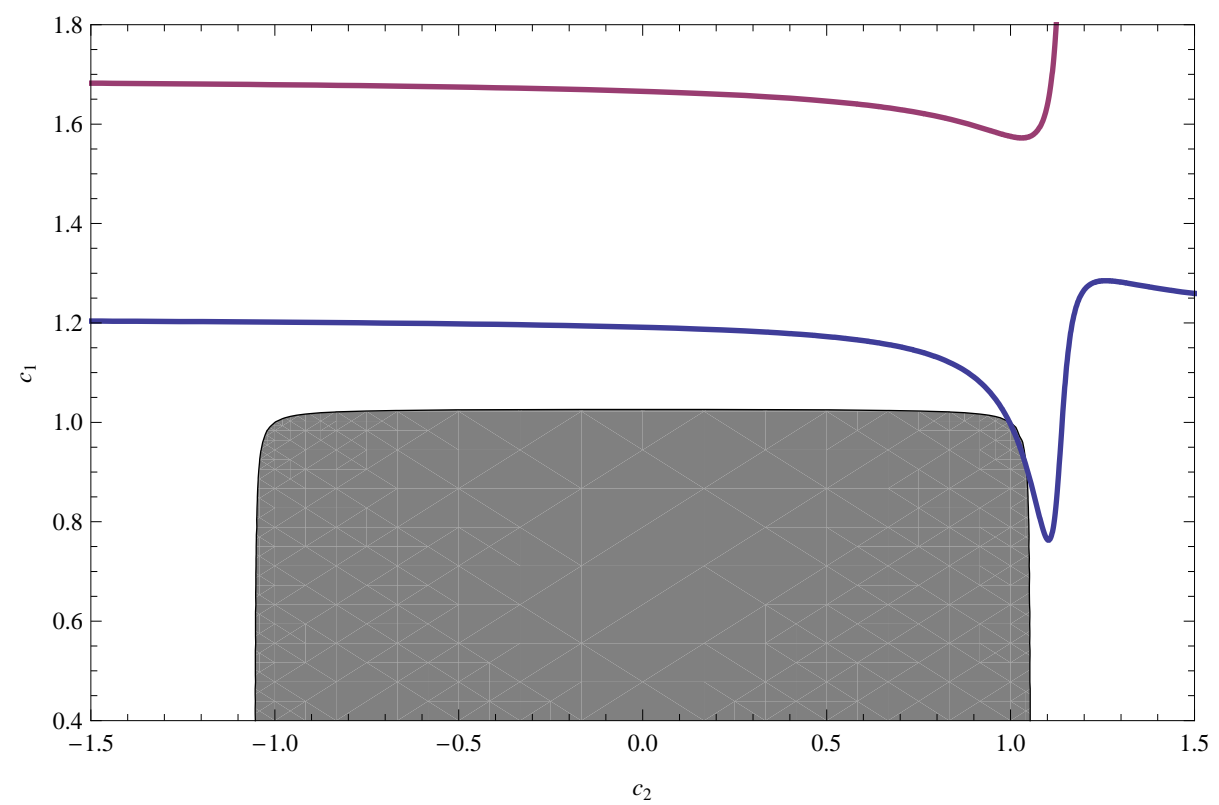

Figure 2. The new-Keynesian model with an alternative parameterization: $\alpha_{1}=0.8, \alpha_{2}=1.05, \iota_{1}=\iota_{2}=0.0, \beta=0.99, \sigma=1.0, \kappa=$ $0.132, p_{11}=0.75$, and $p_{22}=0.95$. The two lines are defined by Equation (24) and the shaded region is defined Inequality (21).

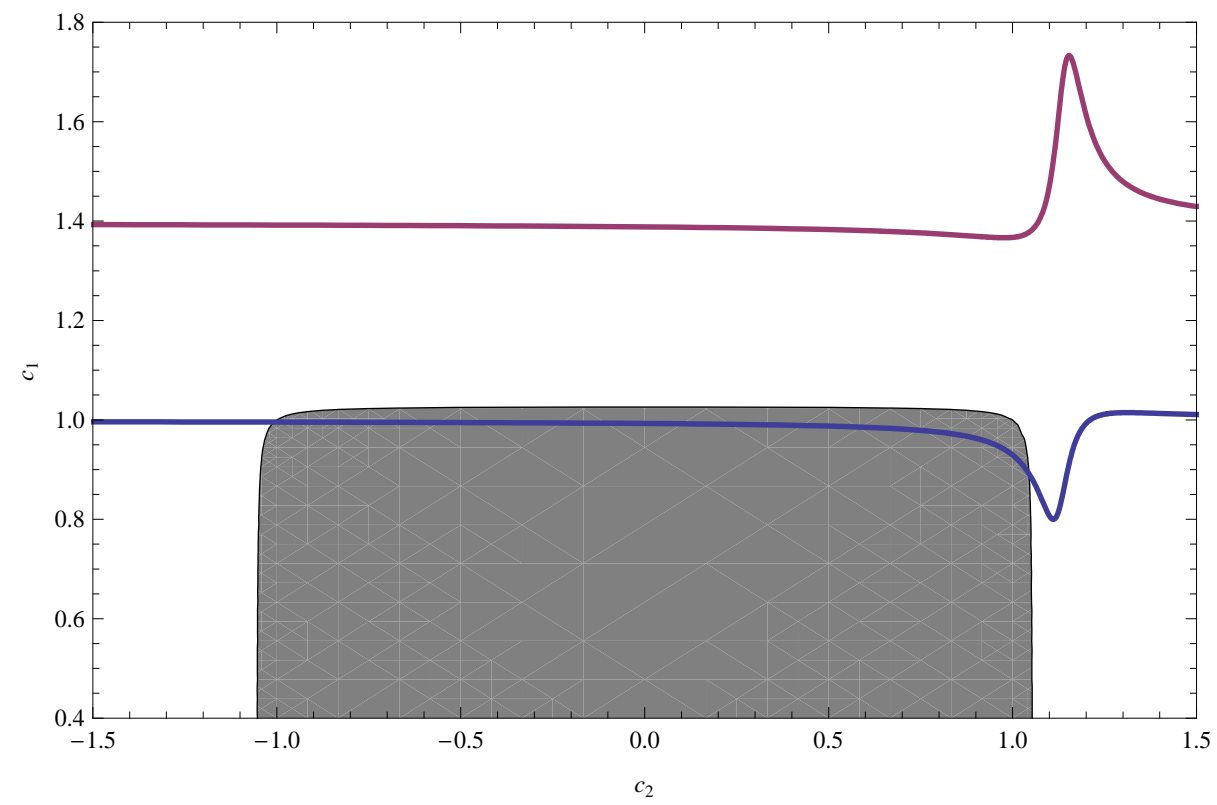

FigurE 3. The new-Keynesian model with an alternative parameterization: $\alpha_{1}=0.8, \alpha_{2}=1.05, \iota_{1}=\iota_{2}=0.0, \beta=0.99, \sigma=1.0, \kappa=$ $0.132, p_{11}=0.90$, and $p_{22}=0.95$. The two lines are defined by Equation (24) and the shaded region is defined Inequality (21). 
persistence of shocks in each regime. Recall that the policy maker in regime 1 follows a passive policy and the policy maker in regime 2 is active.

From the scales on both the $\mathrm{x}$-axis and the $\mathrm{y}$-axis in Figure 3 one can see that there is not much variation in the admissible values of $c_{1}$ while indeterminacy exists for a wide range of $c_{2}$. It follows from the fact that the range of $c_{1}$ inside the indeterminate region is small, that the characteristics of indeterminate dynamics in regime 1 are similar for the entire set of indeterminate equilibria.

There is, however, a wide range of possible values of $c_{2}$ that are consistent with an indeterminate equilibrium. Monetary policy is active in regime 2 , and if regime 2 were an absorbing state, then once the system entered regime 2 the equilibrium would be determinate. But since the system can escape back to the passive regime, indeterminacy may spillover to the active regime and lead to many possible dynamic paths for the state variables in regime 2, even though the Fed follows an active policy. Further, the characteristics of each of these equilibria varies widely.

The parameter $c_{2}$ represents the degree of autocorrelation of the non-fundamental shock in the active regime and Figure 3 shows that this can vary from -1 to a value greater then 1 with every possible value in between. Each of these values represents a different equilibrium with a very different degree of persistence for the observed behavior of inflation, output and the interest rate.

\section{Conclusion}

Our main contribution in this paper was to provide a set of necessary and sufficient conditions for determinacy in a class of forward looking Markov-switching rational expectations models. To accomplish this task, we showed how the question of determinacy of a rational expectations model can be restated as a stability question in a class of Markov-switching models. To make progress, we argued for the use of mean-square stability rather than bounded stability as the appropriate stability concept.

A second important contribution was to show how determinacy can be restated as a constrained optimization problem. This restatement permits an applied researcher to partition the parameter space of an economic model into determinate and indeterminate regions for a large class of Markov-switching rational expectations models and hence, to compute the likelihood for each regime.

Finally, we provided an application of our approach in the context of the familiar new-Keynesian model. For this example, the constrained optimization problem is amenable to a graphical analysis. We believe that our technique will provide useful 
in a wide variety of practical applications and we hope to extend it in future work to the case where the state vector may contain one or more predetermined variables.

\section{Appendix A. Proofs}

Proof of Theorem 1. Let $y_{t}$ be a solution of Equation (1). We must show that $y_{t}$ can be represented by Equations (15) and (16). Define $w_{t}$ by $w_{t}=y_{t}-G_{s_{t}} u_{t}$. By substituting this expression into Equation (1) and making use of the definition

$$
G_{s_{t}} \Gamma_{s_{t}}^{-1} \Psi_{s_{t}}
$$

it follows that the process $w_{t}$ must be a solution of

$$
\Gamma_{s_{t}} w_{t}=E_{t}\left[w_{t+1}\right]
$$

We must show that Equation (A2) holds when $w_{t}$ is represented by Equation (16). Let $V_{i}$ be any matrix with orthonormal columns such that the column space of $V_{i}$ is the span of the support of $w_{t} 1_{\left\{s_{t}=i\right\}}$, where $1_{\left\{s_{t}=i\right\}}$ denotes the indicator function that is one if $s_{t}=i$ and zero otherwise. ${ }^{6}$ Let $k_{i}$ be the dimension of the column space of $V_{i}$. Since $w_{t}$ is a solution of Equation (A2), the following equation holds almost surely.

$$
\begin{aligned}
\Gamma_{i} v & =E\left[\Gamma_{s_{t}} w_{t} \mid w_{t}=v, s_{t}=i\right]=E\left[E_{t}\left[w_{t+1}\right] \mid w_{t}=v, s_{t}=i\right] \\
& =E\left[w_{t+1} \mid w_{t}=v, s_{t}=i\right]=\sum_{j=1}^{h} p_{i, j} E\left[w_{t+1} \mid w_{t}=v, s_{t}=i, s_{t+1}=j\right] .
\end{aligned}
$$

Because the column space of $V_{j}$ is the span of the support of $w_{t+1} 1_{\left\{s_{t+1}=j\right\}}$, it follows that $E\left[w_{t+1} \mid w_{t}=v, s_{t}=i, s_{t+1}=j\right]$ is almost surely in the column space of $V_{j}$. This and the fact that the column space of $V_{i}$ is the span of the support of $w_{t} 1_{\left\{s_{t}=i\right\}}$, implies that there exists a $k_{j} \times k_{i}$ matrix $\Phi_{i, j}$ such that

$$
\Gamma_{i} V_{i}=\sum_{j=1}^{h} p_{i, j} V_{j} \Phi_{i, j}
$$

Define $\gamma_{t}=w_{t}-V_{s_{t}} \Phi_{s_{t-1}, s_{t}} V_{s_{t-1}}^{\prime} w_{t-1}$. Because $w_{t}$, and hence $\gamma_{t}$, is almost surely in the column space of $V_{s_{t}}, \gamma_{t}=V_{s_{t}} V_{s_{t}}^{\prime} \gamma_{t}$. All that remains to be shown is that

\footnotetext{
${ }^{6}$ If the support of $w_{t} 1_{\left\{s_{t}=i\right\}}$ is $\{0\}$, then we take $V_{i}$ to be the $n \times 0$ matrix and follow the usual conventions of dealing with matrices that have a zero dimension.
} 
$E_{t-1}\left[V_{s_{t}} V_{s_{t}}^{\prime} \gamma_{t}\right]=0$. Since

$$
\begin{aligned}
E_{t-1}\left[V_{s_{t}} V_{s_{t}}^{\prime} \gamma_{t}\right] & =E_{t-1}\left[w_{t}-V_{s_{t}} \Phi_{s_{t-1}, s_{t}} V_{s_{t-1}}^{\prime} w_{t-1}\right] \\
& =\Gamma_{s_{t-1}} w_{t-1}-\sum_{j=1}^{h} p_{s_{t}, j} V_{j} \Phi_{s_{t-1}, j} V_{s_{t-1}}^{\prime} w_{t-1} \\
& =\Gamma_{s_{t-1}} w_{t-1}-\Gamma_{s_{t-1}} V_{s_{t-1}} V_{s_{t-1}}^{\prime} w_{t-1} \\
& =0
\end{aligned}
$$

where the last equality holds because $w_{t-1}$ is almost surely in the column space of $V_{s_{t-1}}$. The theorem follows.

Proof of Theorem 2. It is straight forward to verify that any process $y_{t}$ defined by Equations (15) and (16) will be a solution of Equation (1) if and only Equation (17) holds. So, all that remains to be shown is that any process $y_{t}$ defined by Equations (15) and (16) will be MSS if and only if Equation (19) holds.

Since the exogenous process $u_{t}$ is mean-zero and independent of the Markov process $s_{t}$, for any $y_{t}$ given by Equations (15) and (16) we have

$$
E\left[y_{t} y_{t}^{\prime}\right]=E\left[G_{s_{t}} u_{t} u_{t}^{\prime} G_{s_{t}}^{\prime}\right]+E\left[G_{s_{t}} u_{t} \gamma_{t}^{\prime} V_{s_{t}}^{\prime} V_{s_{t}}\right]+E\left[V_{s_{t}} V_{s_{t}}^{\prime} \gamma_{t} u_{t}^{\prime} G_{s_{t}}^{\prime}\right]+E\left[w_{t} w_{t}^{\prime}\right]
$$

Since $u_{t}$ and $\gamma_{t}$ are assumed to be jointly MSS, the first three terms on the right hand side of the above equation will converge as $t$ increases. Thus $y_{t}$ will be MSS if and only if $w_{t}$ is MSS.

We apply Theorem 3.9 and 3.33 of Costa, Fragoso, and Marques (2004, pages 36 and 49) to obtain necessary and sufficient conditions for $w_{t}$ to be MSS. Theorem 3.33 states that Equation (16) defines a MSS process if and only if the homogeneous equation

$$
w_{t}=\Lambda_{s_{t-1}, s_{t}} w_{t-1}
$$

defines a MSS process. Theorem 3.9 states that Equation (A3) defines a MSS process if and only $r_{\sigma}\left(\mathcal{A}_{1}\right)<1$. The matrix $\mathcal{A}_{1}$ has the property that if $\Sigma_{i, t}=E\left[y_{t} y_{t}^{\prime} 1_{\left\{s_{t}=i\right\}}\right]$, where $1_{\left\{s_{t}=i\right\}}$ is the indicator function that is one if $s_{t}=i$, then

$$
\operatorname{vec}\left(\Sigma_{1, t+1}, \cdots, \Sigma_{h, t+1}\right)=\mathcal{A}_{1} \operatorname{vec}\left(\Sigma_{1, t}, \cdots, \Sigma_{h, t}\right)
$$

Since $E\left[y_{t} y_{t}^{\prime}\right]=\sum_{i=1}^{h}\left(\sum_{i, t}\right)$, it is not surprising that the matrix $\mathcal{A}_{1}$ is key in determinining mean-square stability. To explicitly define $\mathcal{A}_{1}$, we first define the following 
two matrices. Let the $h^{2} \times h^{2}$ matrix $\hat{P}$ be

$$
\hat{P}=\begin{gathered}
(1,1) \\
(1,2) \\
\vdots \\
\vdots \\
(\mathrm{h}, 1) \\
(\mathrm{h}, 2) \\
\vdots \\
(\mathrm{h}, \mathrm{h})
\end{gathered} \quad\left[\begin{array}{cccccccccc}
(1,1) & \cdots & (1, \mathrm{~h}) & (2,1) & \cdots & (2, \mathrm{~h}) & \cdots & (\mathrm{h}, 1) & \cdots & (\mathrm{h}, \mathrm{h}) \\
p_{1,1} & \cdots & p_{1, h} & 0 & \cdots & 0 & \cdots & 0 & \cdots & 0 \\
0 & \cdots & 0 & p_{2,1} & \cdots & p_{2, h} & \cdots & 0 & \cdots & 0 \\
\vdots & & \vdots & \vdots & & \vdots & \ddots & \vdots & & \vdots \\
0 & \cdots & 0 & 0 & \cdots & 0 & \cdots & p_{h, 1} & \cdots & p_{h, h} \\
\vdots & & \vdots & \vdots & & \vdots & & \vdots & & \vdots \\
p_{1,1} & \cdots & p_{1, h} & 0 & \cdots & 0 & \cdots & 0 & \cdots & 0 \\
0 & \cdots & 0 & p_{2,1} & \cdots & p_{2, h} & \cdots & 0 & \cdots & 0 \\
\vdots & & \vdots & \vdots & & \vdots & \ddots & \vdots & & \vdots \\
0 & \cdots & 0 & 0 & \cdots & 0 & \cdots & p_{h, 1} & \cdots & p_{h, h}
\end{array}\right] .
$$

The matrix $\hat{P}$ is the transition matrix for the Markov process $\left(s_{t-1}, s_{t}\right)$. Let the $n^{2} h^{2} \times n^{2} h^{2}$ matrix $D\left(\Lambda_{i, j}\right)$ be

$$
D\left(\Lambda_{i, j}\right)=\left[\begin{array}{ccccccc}
\Lambda_{1,1} \otimes \Lambda_{1,1} & \cdots & 0 & \cdots & 0 & \cdots & 0 \\
\vdots & \ddots & \vdots & & \vdots & \ddots & \vdots \\
0 & \cdots & \Lambda_{1, h} \otimes \Lambda_{1, h} & \cdots & 0 & \cdots & 0 \\
\vdots & & \vdots & \ddots & \vdots & & \vdots \\
0 & \cdots & 0 & \cdots & \Lambda_{h, 1} \otimes \Lambda_{h, 1} & \cdots & 0 \\
\vdots & \ddots & \vdots & & \vdots & \ddots & \vdots \\
0 & \cdots & 0 & \cdots & 0 & \cdots & \Lambda_{h, h} \otimes \Lambda_{h, h}
\end{array}\right] .
$$

The matrix $\mathcal{A}_{1}$ is defined to be $\left(\hat{P}^{\prime} \otimes I_{n^{2}}\right) D\left(\Lambda_{i, j}\right)$. To complete the proof, we show that the non-zero eigenvalues of $\mathcal{A}_{1}$ are the same as the non-zero eigenvalues of $M_{1}\left(\Phi_{i, j}\right)$, which implies that $r_{\sigma}\left(\mathcal{A}_{1}\right)<1$ if and only $r_{\sigma}\left(M_{1}\left(\Phi_{i, j}\right)\right)<1$.

Suppose that the $h^{2} n^{2}$-dimensional vector $\left(v_{1,1}, \cdots, v_{1, h}, \cdots, v_{h, 1}, \cdots, v_{h, h}\right)$ is an eigenvector of $\left(\hat{P}^{\prime} \otimes I_{n^{2}}\right) D\left(\Lambda_{i, j}\right)$ with eigenvalue $\lambda \neq 0$. This implies that

$$
p_{i, j} \sum_{k=1}^{h}\left(\Lambda_{k, i} \otimes \Lambda_{k, i}\right) v_{k, i}=\lambda v_{i, j}
$$

Define $v_{i}=\sum_{k=1}^{h}\left(\Lambda_{k, i} \otimes \Lambda_{k, i}\right) v_{k, i}$, which implies that $v_{i, j}=\frac{p_{i, j}}{\lambda} v_{i}$. Substituting this into Equation (A4) gives $\frac{p_{i, j}}{\lambda} \sum_{k=1}^{h} p_{k, i}\left(\Lambda_{k, i} \otimes \Lambda_{k, i}\right) v_{k}=p_{i, j} v_{i}$. Since for every $i$ there is at least one $j$ such that $p_{i, j} \neq 0$, this implies that $\sum_{k=1}^{h} p_{k, i}\left(\Lambda_{k, i} \otimes \Lambda_{k, i}\right) v_{k}=\lambda v_{i}$, which is precisely the condition needed for the $h n^{2}$-dimensional vector $\left(v_{1}, \cdots, v_{h}\right)$ to be an eigenvector of $M\left(\Lambda_{i, j}\right)$ with eigenvalue $\lambda$. Reversing this argument shows that any 
eigenvalue of $M\left(\Lambda_{i, j}\right)$ will also be an eigenvalue of $\mathcal{A}_{1}$. Thus the non-zero eigenvalues of $\mathcal{A}_{1}$ are the same as the non-zero eigenvalues of $M_{1}\left(\Lambda_{i, j}\right)$. Finally, because

$$
M_{1}\left(\Lambda_{i, j}\right)=\operatorname{diag}\left(V_{i} \otimes V_{i}\right) M_{1}\left(\Phi_{i, j}\right) \operatorname{diag}\left(V_{i}^{\prime} \otimes V_{i}^{\prime}\right)
$$

the non-zero eigenvalues of $M_{1}\left(\Lambda_{i, j}\right)$ are the same as the non-zero eigenvalues of $M_{1}\left(\Phi_{i, j}\right)$.

Two points need to be made about the assumptions in Theorems 3.9 and 3.33 of of Costa, Fragoso, and Marques (2004, pages 36 and 49). First, the derivation of these theorems are in the complex-valued case. There are some subtleties when applying these derivations to the real-valued case concerning the permissible initial values. The details of this are worked out in ? and both of these Theorems hold in the realvalued case. Second, in Theorem 3.33 the exogeneous shocks $\gamma_{t}$ are assumed to be an independent covariance stationary process independent of the Markov process $s_{t}$ and the initial condition $w_{0}$. However, in the proofs all we really need is that $E\left[\gamma_{t}\right] \sim$ $\mu_{\gamma}, E\left[\gamma_{t} \gamma_{t}^{\prime}\right] \sim \Sigma_{\gamma}$, and $E\left[\gamma_{t} 1_{\left\{s_{t}=j\right\}} w_{t}^{\prime} 1_{\left\{s_{t-1}=i\right\}}\right]=0$, where $\mu_{\gamma}$ is any $n$-dimensional vector, $\Sigma_{\gamma}$ is any $n \times n$ symmetric and positive semi-definite matrix, and $1_{\left\{s_{t}=j\right\}}$ and $1_{\left\{s_{t-1}=i\right\}}$ are indicator functions. In contrast, we assume that the $\gamma_{t}$ are a mean-zero MSS process independent of the Markov process $s_{t}$, which implies that $E\left[\gamma_{t}\right]=0$, $E\left[\gamma_{t} \gamma_{t}^{\prime}\right] \sim \Sigma_{\gamma}$, and $E\left[\gamma_{t} 1_{\left\{s_{t}=j\right\}} w_{t}^{\prime} 1_{\left\{s_{t-1}=i\right\}}\right]=0$ as required.

Proof of Proposition 1. If we define $V_{i}=v_{i} /\left\|v_{i}\right\|$ and $\Phi_{i, j}=\left\|v_{j}\right\| c_{i} /\left\|v_{i}\right\|$, then Equation (17) can be written in matrix form as

$$
\left(\left[\begin{array}{ccc}
\Gamma_{1} & \cdots & 0 \\
\vdots & \ddots & \vdots \\
0 & \cdots & \Gamma_{h}
\end{array}\right]-\left[\begin{array}{ccc}
p_{1,1} \Phi_{1,1} & \cdots & p_{1, h} \Phi_{1, h} \\
\vdots & \ddots & \vdots \\
p_{h, 1} \Phi_{h, 1} & \cdots & p_{h, 1} \Phi_{h, 1}
\end{array}\right]\right)\left[\begin{array}{c}
v_{1} /\left\|v_{1}\right\| \\
\vdots \\
v_{h} /\left\|v_{h}\right\|
\end{array}\right]=0
$$

which is equivalent to Equation (20). Thus, by Theorem 2, the solution given by Equations (22) and (23) will be MSS if and only if $r_{\sigma}\left(M_{1}\left(\Phi_{i, j}\right)\right)<1$. Since

$$
M_{1}\left(\Phi_{i, j}\right)=\operatorname{diag}\left(\left\|v_{i}\right\|^{2}\right)\left(\operatorname{diag}\left(c_{i}^{2}\right) P\right) \operatorname{diag}\left(\left\|v_{i}\right\|^{2}\right)^{-1}
$$

$r_{\sigma}\left(M_{1}\left(\Phi_{i, j}\right)\right)=r_{\sigma}\left(\operatorname{diag}\left(c_{i}^{2}\right) P\right)$. This completes the proof of the first two parts of the proposition.

To prove the third part, note that the process $y_{t}$ given by Equations (15) and (16) will be bounded if and only the process $w_{t}$ given by Equation (16) is bounded. Since 


$$
\begin{aligned}
\Lambda_{s_{t-1}, s_{t}}=c_{s_{t-1}} v_{s_{t}} v_{s_{t-1}}^{\prime} /\left\|v_{s_{t-1}}\right\|^{2} & \\
\left\|w_{t}\right\| & =\left\|v_{s_{t}}\right\|\left|\prod_{i=1}^{t} c_{s_{i-1}} \frac{v_{s_{0}}^{\prime} w_{0}}{\left\|v_{s_{0}}\right\|^{2}}+\sum_{i=2}^{t}\left(\prod_{j=i}^{t} c_{s_{j-1}}\right) v_{s_{i-1}}^{\prime} \gamma_{i-1}+v_{s_{t}}^{\prime} \gamma_{t}\right| \\
& \leq\left\|v_{s_{t}}\right\|\left(\sum_{i=0}^{t} c^{i} a\right)
\end{aligned}
$$

where $c=\max \left\{\left|c_{1}\right|, \cdots,\left|c_{h}\right|\right\}$ and $a=\sup \left\{\left|v_{s_{t}}^{\prime} \gamma_{t}\right|,\left|v_{s_{0}}^{\prime} w_{0}\right| /\left\|v_{s_{0}}\right\|^{2}\right\}$. Thus the $w_{t}$, and hence the $y_{t}$, will be bounded if $\left|c_{i}\right|<1$ for all $i$.

On the other hand, if $\left|c_{i}\right| \geq 1$ for some $i$, then for as long as the Markov process remains in state $i, w_{t}$ will grow exponentially $\left(\left|c_{i}\right|>1\right)$ or follow a random walk $\left(\left|c_{i}\right|=1\right)$. Since the Markov process can remain in state $i$ for arbitrarily long periods of time, the process $w_{t}$, and hence the process $y_{t}$, cannot be bounded. 


\section{REFERENCES}

Blake, A. P., And F. Zampolli (2006): "Optimal Monetary Policy in Markov-

Switching Models with Rational Expectations Agents," Bank of England Working Paper No. 298.

Clarida, R., J. Galí, and M. Gertler (2000): "Monetary Policy Rules and Macroeconomic Stability: Evidence and Some Theory," Quarterly Journal of Economics, CXV, 147-180.

Cogley, T., and T. J. Sargent (2002): "Evolving US Post-Wolrd War II Inflation Dynamics," NBER Macroeconomics Annual, 16, 331-373.

(2005): "Drifts and Volatilities: Monetary Policies and Outcomes in the Post WWII U.S.," Review of Economic Dynamics, 8, 262-302.

Costa, O., M. Fragoso, and R. Marques (2004): Discrete-Time Markov Jump Linear Systems. Springer, New York.

Davig, T., And E. M. Leeper (2006): "Fluctuating Macro Policies and the Fiscal Theory," in NBER Macroeconomic Annual 2006, ed. by D. Acemoglu, K. Rogoff, and M. Woodford. MIT Press, Cambridge, MA.

(2007): "Generalizing the Taylor Principle," American Economic Review, $97(3), 607-635$.

Farmer, R. E., D. F. Waggoner, and T. Zha (2008a): "Generalizing the Taylor Principle: A Comment," American Economic Review, Forthcoming.

- (2008b): "Indeterminacy in a Forward Looking Regime Switching Model," International Journal of Economic Theory, Forthcoming.

- (2008c): "Minimal State Variable Solutions to Markov-Switching Rational Expectations Models," Federal Reserve Bank of Atlanta Working Paper 2008-23.

Hamilton, J. D. (1989): "A New Approach to the Economic Analysis of Nonstationary Time Series and the Business Cycle," Econometrica, 57(2), 357-384.

Leeper, E. M. (1991): 'Equilibria under 'Active' and 'Passive' Monetary and Fiscal Policies," Journal of Monetary Economics, 27, 129-147.

Leeper, E. M., And T. ZhA (2003): "Modest Policy Interventions," Journal of Monetary Economics, 50(8), 1673-1700.

LiU, Z., D. F. WAGgoner, And T. Zha (2008): "Asymmetric Expectation Effects of Regime Shifts in Monetary Policy," Review of Economic Dynamics, Forthcoming.

Lubik, T. A., And F. Schorfheide (2003): "Computing Sunspot Equilibria in Linear Rational Expectations Models," Journal of Economic Dynamics $\&$ Control, $28,273-285$. 
_ (2004): "Testing for Indeterminacy: An Application to U.S. Monetary Policy," American Economic Review, 94(1), 190-219.

McCallum, B. T. (1983): "On Non-Uniqueness in Rational Expectations Models: An Attempt at Perspective," Journal of Monetary Economics, 11, 139-168.

Sims, C. A., And T. Zha (2006): "Were There Regime Switches in US Monetary Policy?," American Economic Review, 96, 54-81.

Svensson, L. E., And N. Williams (2005): "Monetary Policy with Model Uncertainty: Distribution Forecast Targeting," Manuscript, Princeton University.

UCla, Federal Reserve Bank of Atlanta, Federal Reserve Bank of Atlanta and EMORY UNIVERSITY 